\title{
Fluorescence dynamics of graphene quantum dots for detecting lard substance
}

\begin{abstract}
Graphene Quantum Dots (GQD) is used for detecting lard substance. It is discovered that the fluorescence for a GQD with a size approximately $5 \mathrm{~nm}$ in size will have a peak at $675 \mathrm{~nm}$. Introducing lard substance to the GQD will induce a broad fluorescence spectrum at the range of 415 till $715 \mathrm{~nm}$. Higher fluorescence is observed from $760 \mathrm{~nm}$ till $860 \mathrm{~nm}$ showing the dynamics fluorescence changes when lard is applied. These fluorescence dynamics when lard is introduced is due to the functional groups of Carbon-Carbon interaction between GQD and lard.
\end{abstract}

Keyword: Fluorescence; Graphene quantum dots; Lard 